DOI: $10.25100 /$ pfilosofica.v0i53.11436

\title{
MONSTRUOS SEMÁNTICOS: EL EMPIRISMO DE CONCEPTOS Y SU ABORDAJE DEL CONTENIDO INTENCIONAL
}

Dalila Serebrinsky

Universidad de Buenos Aires, Argentina

\begin{abstract}
Resumen
El empirismo de conceptos propuesto por Jesse Prinz pretende ser una teoría de conceptos que cumpla con todos los desiderata que, según el mismo autor, una teoría de conceptos debe cumplir y, a la vez, lo haga de la manera más económica posible. Con respecto al contenido intencional, propone una semántica informacional no atomista. Los conceptos, para Prinz, son meros detectores de rasgos perceptibles, es decir, entidades estructuradas, cuyas partes significativas obtienen su contenido de manera informacional. Es el objetivo de este trabajo mostrar que el empirismo de conceptos es incapaz de satisfacer el desideratum que se refiere a la explicación del contenido intencional. Argumentamos que los conceptos entendidos como meros detectores no logran dar cuenta satisfactoriamente del contenido de los conceptos sin caer en el problema de la disyunción y que son incompatibles con el externalismo propio de las teorías informacionales.
\end{abstract}

Palabras clave: empirismo de conceptos; contenido intencional, detectores; semántica informacional; atomismo semántico.

Cómo citar este artículo: Serebrinsky, D. (2021). Monstruos semánticos: el empirismo de conceptos y su abordaje del contenido intencional. Praxis Filosófica, (53), 31-52. https://doi.org/10.25100/ pfilosofica.v0i53.11436

Recibido: 22 de enero de 2021. Aprobado: 23 de abril de 2021. 


\title{
Semantic Monsters: Concept Empiricism and its Approach to Intentional Content
}

\author{
Dalila Serebrinsky ${ }^{1}$
}

\begin{abstract}
Concept empiricism, introduced by Jesse Prinz, intends to be a theory about concepts which satisfies the desiderata that, according to that author, every theory of concepts must fulfill, and which does it as economically as possible. Regarding intentional content, it puts forward a non atomistic informational semantic theory. According to Prinz, concepts are merely detectors for perceptible features, that is, they are structured entities. The meaningful parts of these detectors obtain their content in an informational way. The aim of this paper is to show that concept empiricism is unable to fulfill the desideratum about intentional content. We argue that concepts as mere detectors are unable to properly account for the content of concepts without facing the disjunction problem, and that they are incompatible with the externalism that characterise informational theories of content.
\end{abstract}

Keywords: Concept Empiricism; Intentional Content; Detectors; Informational Semantics; Semantic Atomism.

${ }^{1}$ Doctoranda en Filosofía de la Universidad de Buenos Aires. Profesora en Filosofía por la Universidad de Buenos Aires. Principal publicación: Serebrinsky, D. y Borge, B. Términos teóricos y teorías híbridas de la referencia (en prensa). Áreas de trabajo: Filosofía de las Ciencias y Filosofía del Lenguaje.

ORCID: 0000-0002-9923-3060 E-mail: dalila.r.ser@gmail.com 


\title{
MONSTRUOS SEMÁNTICOS: EL EMPIRISMO DE CONCEPTOS Y SU ABORDAJE DEL CONTENIDO INTENCIONAL
}

\author{
Dalila Serebrinsky \\ Universidad de Buenos Aires, Argentina
}

\section{Introducción}

Es comúnmente aceptado que nuestros estados mentales están integrados por conceptos. Los estados que se considera que constituyen el pensamiento, como las actitudes proposicionales, están formados por conceptos y obtienen su contenido gracias al contenido de estos. Por lo tanto, dar con una teoría adecuada y completa acerca de qué tipo de estado son estas representaciones y cómo funcionan resulta de vital importancia para la filosofía de la mente y de la psicología.

En Furnishing the Mind: Concepts and their Perceptual Basis (2002), Jesse Prinz lista los desiderata que tal teoría debería cumplir. Una teoría de conceptos debe tener el alcance suficiente para incluir a todas las representaciones que consideramos conceptos, a la vez que debe ser lo suficientemente restrictiva para excluir a aquellos estados que claramente no lo son. Además, esta teoría debería ser capaz de dar cuenta tanto del contenido intencional como del contenido cognitivo de los conceptos. También es deseable que una teoría tal dé cuenta de ciertas características que quisiéramos atribuir a los conceptos, como composicionalidad y publicidad. Finalmente, esta teoría deberá ser compatible con lo que sabemos de ciertos fenómenos que se observan en los agentes con respecto a la adquisición de conceptos y a las tareas de categorización que los involucran. Además de 
esta lista, Prinz ofrece lo que pretende ser una teoría que satisfaga todas estas condiciones: el empirismo de conceptos.

El objetivo de este trabajo es mostrar que el empirismo de conceptos es incapaz de cumplir con algunos de los desiderata mencionados. En particular, es insuficiente para dar cuenta del contenido intencional. La teoría de Prinz pretende explicar este rasgo de los conceptos identificándolos con entidades estructuradas que obtienen su contenido de manera informacional, es decir, por un mecanismo causal. Pero las teorías causales del contenido tienen ciertos rasgos que no son compatibles con el tipo de vehículos que propone el empirismo. En particular, argumentaremos que esa concepción de los conceptos impide ofrecer una salida al problema de la disyunción y que es incapaz de conjugarse con el externalismo típico de las teorías causales. Así, mostraremos que la propuesta de Prinz para este tipo de representaciones tiene los mismos resultados que Fodor le adjudicó a otras formas de semántica informacional no atomista: si bien es posible formularla, "es antinatural y engendra monstruos" (Fodor, 1994, p. 7).

En la segunda sección, haremos una breve exposición del empirismo de conceptos, tal como fuera presentado por Prinz. Nos detendremos en su propuesta semántica y el papel que juega en ella la distinción entre detectores e indicadores. En la tercera sección, describiremos las teorías causales del contenido mental, los desafíos que deben enfrentar y algunas de sus consecuencias. El cuarto apartado estará dedicado a mostrar que la teoría semántica ofrecida por el empirismo de conceptos no es capaz de resolver los problemas que una teoría causal del contenido plantea. Finalmente, en la quinta sección presentaremos las conclusiones.

\section{EI empirismo de conceptos.}

\section{II.1 Los vehículos conceptuales}

Prinz (2002) sostiene una posición empirista respecto a los conceptos y propone que estos sean entendidos como representaciones cuyo vehículo es del mismo tipo que las representaciones que tienen su origen en la percepción. Los conceptos, para Prinz, serían copias o combinaciones de copias de representaciones perceptivas. Las representaciones perceptivas, a su vez, son aquellas que provienen de los sentidos, entendiendo a estos como sistemas de inputs dedicados. Son sistemas de inputs, porque reciben información de alguna parte del entorno extramental, es decir, del propio cuerpo o del entorno exterior. Son dedicados, porque cada sistema procesa inputs que pertenecen a una determinada magnitud. Es decir, que un mismo sistema no procesa inputs de magnitudes diferentes. Las representaciones perceptivas, según esta propuesta, son modales, por lo que cada sistema, 
además de responder a un tipo particular de inputs, genera un tipo particular de representaciones. Los conceptos, entonces, no son representaciones amodales, sino que son representaciones multimodales, ya que mantienen la modalidad de las representaciones perceptivas de las cuales son copia.

Los conceptos no difieren de los estados perceptivos más que en el hecho de que pueden activarse voluntariamente y en ausencia del estímulo. Según el empirismo de conceptos, las distintas representaciones perceptivas de objetos que poseen alguna propiedad son almacenadas en la memoria en una red, la red que corresponde al concepto que refiere a esa propiedad. Cuando pensamos en esa propiedad y necesitamos el concepto en cuestión, activamos en la memoria de trabajo una representación que actúa en reemplazo de toda la red. Esa representación es llamada proxytipo (proxytype) (Prinz, 2002, p. 149). Un proxytipo es una representación perceptiva concreta que queda almacenada como parte de la red correspondiente a un concepto. Pero no es un tipo de representación en particular, sino que cualquier representación puede actuar como proxytipo, ya que la selección del proxytipo a utilizar como vehículo del concepto está determinada por el contexto.

El fundamento principal de esta propuesta, y su mayor atractivo, es su carácter económico. En efecto, el empirismo de conceptos requiere de menos tipos de entidades que otras teorías de conceptos y disuelve algunas de las distinciones entre representaciones a las que muchas teorías del campo suscriben, como la distinción conceptual/ no conceptual ${ }^{2}$ o la distinción modal/amodal ${ }^{3}$.

\section{2 Detectores e indicadores}

El empirismo de conceptos que describimos pretende comprometerse con una teoría informacional para dar cuenta del contenido intencional de los conceptos. La teoría informacional (Dretske, 1981) sostiene que un tipo estado porta información acerca de otro tipo de estado si las instancias de ambos tipos covarían nomológicamente. Entonces, el contenido de los

\footnotetext{
${ }^{2}$ Evans (1982) introdujo esta distinción al proponer que no todos nuestros estados mentales están constituidos por conceptos, sino que existe otro tipo de estados: los estados no conceptuales. La distinción es ya tradicional en la filosofía de la mente y de las ciencias cognitivas, y algunas de sus motivaciones provienen de la necesidad de explicar la experiencia perceptiva y el pensamiento en criaturas no lingüísticas o pre-lingüísticas. Para un argumento a favor de la tesis de que el empirismo de conceptos disuelve esta distinción, ver Skidelsky (2011).

${ }^{3}$ Se dice que una representación es modal cuando está codificada como la información que corresponde a algún sistema perceptivo específico (podrá tener una modalidad visual, auditiva, etc.) y que es amodal cuando el tipo de código que emplea no pertenece a ninguno de esos tipos. Para un argumento a favor de la tesis de que el empirismo de conceptos disuelve esta distinción y una crítica, ver Markman y Stilwell (2004).
} 
conceptos estaría determinado por la información que lleva acerca de la propiedad que representan, es decir, por la propiedad con cuyas instancias covaría nomológicamente.

La semántica informacional es comúnmente asociada al atomismo, es decir, a la posición según la cual el significado de un signo no está determinado por su relación con ningún otro ítem significativo. Esto es lo que sostiene una teoría de conceptos como, por ejemplo, la de Fodor (1998), que describe los conceptos a la manera informacional, como tipos de representaciones cuyos casos están enlazados de manera fiable con instancias de alguna propiedad. Pero Fodor sostiene, además, que los conceptos son representaciones atómicas, es decir, que no están estructurados, por lo que su contenido está determinado por nada más que aquella propiedad con la que cada uno covaría y es independiente del contenido de otros conceptos. El atomismo resulta del rechazo al holismo, al advertir que una semántica holista traería problemas para explicar la adquisición de conceptos y no podría dar cuenta de dos de las características que toda teoría de conceptos debe explicar: la composicionalidad y la publicidad. Prinz, en cambio, intenta ofrecer una explicación del contenido dentro de su empirismo de conceptos que considera las representaciones como estructuradas, a la vez que atribuye su contenido a relaciones con el mundo como las descriptas en una teoría informacional.

Para exponer su teoría del contenido intencional de los conceptos, Prinz recurre a la distinción entre indicadores y detectores. Un indicador es una entidad que covaría con una propiedad del mundo. Un detector, en cambio, es el mecanismo que media entre esa entidad y esa propiedad. Tanto los indicadores como los detectores portan información, pero los primeros son no estructurados, mientras que los detectores pueden tener estructura interna. Cada detector puede tener partes significativas a las cuales reacciona (Prinz, 2002, pp. 123-124). Entonces, el detector que permite indicar, por ejemplo, el numeral 5, tendrá una parte que detecta curvas, otra que detecta líneas rectas, otra que detecta cierto tipo de ángulo, etc. La detección de estos rasgos permitirá al detector reaccionar y, ante la presencia del numeral 5 , activar el indicador para ese símbolo. A la vez, y gracias a esa estructura, el detector mencionado será más similar al detector para, por ejemplo, el numeral 2 que al detector para el numeral 4 .

En el marco de otras teorías de conceptos, los detectores serían los mecanismos que recogen los rasgos perceptibles de los objetos gracias a los cuales se activa el concepto (el indicador) correspondiente. Pero, para el empirismo de conceptos, esta explicación cuenta con un tipo de entidad en exceso. Prinz propone que los conceptos sean identificados, no con los 
indicadores, sino con los detectores. Por un lado, si hay una relación causal entre los mecanismos perceptivos de detección y la activación de conceptos, entonces éstos deben ser estados perceptivos. Por otro lado, la identificación de los conceptos con detectores significa atribuirles estructura, lo cual tiene ventajas explicativas con respecto a algunos fenómenos, como los de adquisición y categorización (Prinz, 2002, p. 124). El contenido de los detectores, además, es informacional, lo cual garantiza una relación causal entre propiedades externas y contenido. Así, el empirismo de conceptos, a diferencia de otros empirismos, no cae en el fenomenalismo (Prinz, 2002, p. 126). Los conceptos entendidos como detectores no sólo combinarían las ventajas de la estructura con las de una teoría informacional, sino que permitirían deshacerse de los indicadores, los cuales serían un tipo de entidad prescindible a la hora de explicar su contenido intencional.

\section{Teorías causales del contenido}

La teoría informacional es un tipo de teoría causal del contenido. Estas teorías sostienen que el contenido de una representación está determinado por aquello que la causa. De esta manera, una representación $\mathrm{R}$ tendrá el contenido $\mathrm{S}$ si y sólo si situaciones del tipo $\mathrm{S}$ causan ocurrencias de representaciones $\mathrm{R}$. Como vimos, la teoría informacional toma este tipo de explicación y le agrega una restricción nomológica: una representación $\mathrm{R}$ tendrá el contenido S si porta información acerca de S, y esto ocurre si y sólo si las instancias de $\mathrm{R}$ se correlacionan nomológicamente con instancias de $\mathrm{S}$, es decir, si y sólo si las ocurrencias de $\mathrm{R}$ covarían de manera fiable con instancias de $\mathrm{S}$, soportando contrafácticos.

\section{III.1 Representaciones erradas y el problema de la disyunción}

Describimos el tipo de relación que para una teoría causal determina el contenido de un signo. Sin embargo, no todos los signos que portan información acerca del estado que los causa pueden ser considerados representaciones. Pensemos, por ejemplo, en los anillos que se forman en los troncos de los árboles y que portan información acerca de su edad. Este tipo de relación cumple con los requisitos de la determinación del contenido expuesta: los anillos covarían con el número de años que cuenta el árbol fiablemente. Además, lo hacen nomológicamente, soportando contrafácticos. Sin embargo, no puede decirse que los anillos representen la edad del árbol. Los anillos del tronco del árbol son un caso de significado natural y el contenido de un estado mental es lo que se llama significado no natural, donde el primer tipo de significado implica la existencia del estado 
de cosas que el signo indica, mientras que el segundo tipo de significado no lo hace (Grice, 1957).

Cuando hablamos de lo que un estado con contenido representa estamos hablando de sus condiciones de verdad, o de corrección. El contenido de una representación, entonces, es aquello que, de ser satisfecho, la haría correcta. Pero sólo tiene sentido hablar de un estado correcto si existe la posibilidad de una representación incorrecta. Es decir, debe existir la posibilidad del error. Eso es precisamente lo que no encontramos en la relación entre el número de anillos en el tronco de un árbol y su número de años. Estas variables siempre se correlacionan en una función identidad, el árbol no puede cometer un error al generar sus anillos. Lo mismo ocurre con el humo que indica la presencia de fuego o las manchas que indican la presencia de una enfermedad. En todos estos casos, existe una relación causal entre la situación indicada por el signo y el signo, pero no hay contenido. Pero nuestros estados mentales intencionales sí son representacionales, ya que tienen condiciones de corrección. Pueden ser semánticamente evaluados como correctos o incorrectos. Si es característico de lo mental que los estados intencionales sean acerca de estados extramentales, entonces es una característica igualmente definitoria el hecho de que estos estados puedan estar errados.

Pero las teorías causales no tienen éxito a la hora de explicar las representaciones erradas. Si el contenido de una representación es aquello que la causa, entonces ésta no podría ser incorrecta. Imaginemos una representación R que se correlaciona causalmente con casos de S. Podríamos decir que $\mathrm{R}$ representa $\mathrm{S}$. Pero imaginemos ahora que algunos casos de $\mathrm{R}$ son causados por casos no de $\mathrm{S}$, sino de $\mathrm{T}$. Deberíamos decir, entonces, que $\mathrm{R}$ representa también $\mathrm{T}$, ya que un estado representa aquello que lo causa. El contenido de R no es ya 'S', sino la disyunción 'S o T', ya que las instancias de $\mathrm{R}$ se correlaciona causalmente tanto con instancias de $\mathrm{S}$ como de $\mathrm{T}$. Pero, si su contenido es la disyunción entre $\mathrm{S}$ y $\mathrm{T}$, entonces la aparición de $\mathrm{R}$ ante un caso de $\mathrm{T}$ no conlleva un error. En ese caso, la ocurrencia de $\mathrm{R}$ es causada por algo en el mundo que hace verdadero su contenido: un caso de T. Decir, además, que la representación en cuestión es causada por algo que hace verdadero su contenido es superfluo, ya que según una explicación causal aquello que causa la representación es lo que determina su contenido, es decir, lo que determina qué hace correcta a la representación en cuestión. El surgimiento de las teorías causales del contenido ha sido también el surgimiento del difícil desafío de explicar el error en las representaciones. Es el desafío de toda teoría causal ofrecer una salida a este problema. 
Dretske (1981) ofreció una explicación de las representaciones erradas, proponiendo que el contenido de una representación está dado por aquello que causó sus instancias en el período de aprendizaje. Cada poseedor del concepto R habría pasado por un período de aprendizaje, en el cual fue garantizado que las ocurrencias de $\mathrm{R}$ sean causadas sólo por instancias de $\mathrm{S}$. Una vez pasado ese período, el poseedor de $\mathrm{R}$ podría toparse con instancias de $\mathrm{T}$ y tener, a causa de ellas, casos de estados R. Pero estos casos no determinan el contenido de $\mathrm{R}$, el cual fue determinado durante el período de aprendizaje como ' $\mathrm{S}$ '. Esas ocurrencias de R, entonces, estarían erradas, ya que su contenido es diferente de aquello que las causa.

Fodor, en su célebre artículo Semantics, Wisconsin Style (1984), denominó problema de la disyunción al problema de las teorías causales para dar cuenta del error. En el mismo artículo, objetó la solución propuesta por Dretske, ya que, según la teoría causal del contenido mental, éste está determinado por la correlación entre el estado y su causa, correlación que soporta contrafácticos. Entonces, podemos suponer que durante el aprendizaje del concepto $\mathrm{R}$ el aprendiz no se encontró con instancias de $\mathrm{T}$. Pero incluso en ese caso, sería correcto decir que si el aprendiz se hubiera encontrado con instancias de T, entonces éstas habrían causado el estado $\mathrm{R}$ en él. Entonces, el problema no está saldado, pues tanto instancias de $\mathrm{S}$ como de $\mathrm{T}$ causarían $\mathrm{R}$ en el aprendiz de serles presentadas, lo cual nos deja nuevamente con un contenido disyuntivo para $\mathrm{R}$.

Fodor (1987), a su vez, propuso la Teoría de la Dependencia Asimétrica como salida al problema de la disyunción. El error que se da cuando un estado R con contenido ' $\mathrm{S}$ ' es causado por un estado $\mathrm{T}$ se explicaría por una relación asimétrica entre el vínculo causal entre instancias de $\mathrm{S}$ e instancias de $\mathrm{R}$ y el vínculo causal entre instancias de $\mathrm{T}$ e instancias de $\mathrm{R}$. El error sería posible porque la relación causal entre instancias de $\mathrm{T}$ e instancias de $\mathrm{R}$ depende de la relación causal entre instancias de $\mathrm{S}$ e instancias de $\mathrm{R}$. Si no existiera la última relación, no podría darse la primera. La dependencia es asimétrica, ya que no se da la misma relación en la dirección opuesta: las instancias de $\mathrm{S}$ causarían instancias de $\mathrm{R}$ incluso si las instancias de $\mathrm{T}$ no lo hicieran.

\section{III.2 Externalismo}

Las teorías causales en general se comprometen con el externalismo respecto del contenido mental. Esta posición sostiene que el contenido de los estados mentales no está determinado por sus propiedades intrínsecas, sino por sus relaciones con objetos y eventos del mundo. Si lo que confiere contenido a un estado es su relación causal con una entidad extramental, como sostienen las 
teorías causales, entonces el contenido no puede provenir de las propiedades intrínsecas del estado.

El clásico argumento a favor del externalismo es el experimento mental de las Tierras gemelas (Putnam, 1975) ${ }^{4}$. Dicho experimento mental consiste en lo siguiente: imaginemos a Oscar, quien vive en la Tierra y tiene el concepto AGUA que refiere al agua, es decir, a la sustancia cuya composición química es $\mathrm{H}_{2} \mathrm{O}$. Pero, además, imaginemos que Oscar tiene un gemelo idéntico, al que llamaremos Oscar $_{\mathrm{g}}$. Oscar ${ }_{\mathrm{g}}$ es idéntico a Oscar en todas sus propiedades, incluyendo sus propiedades mentales, es decir, que cuenta con los mismos estados estrictos (narrow) que Oscar. Pero Oscar ${ }_{g}$ vive en la Tierra Gemela, un planeta que es exactamente como la Tierra, con la excepción de que, en lugar de agua, hay una sustancia a la que podemos llamar agua $a_{\mathrm{g}}$ la cual tiene una composición química muy compleja que abreviamos mediante la fórmula XYZ. Esta sustancia tiene las mismas propiedades fenoménicas que el agua y, además, ocupa los mismos lugares y cumple las mismas funciones en la Tierra Gemela que aquellos que el agua ocupa y cumple en la Tierra. En la Tierra Gemela, además, no hay agua. La intuición que pretende activar este experimento mental es la de que Oscar ${ }_{\mathrm{g}}$, a pesar de ser absolutamente igual a Oscar en sus propiedades intrínsecas, no tiene los mismos conceptos que él: Oscar tiene el concepto AGUA mientras que Oscar ${ }_{\mathrm{g}}$ no lo tiene, puesto que nunca se ha encontrado con el agua. Esto es así porque el contenido mental, para el externalista, está determinado en parte por aquello en el mundo con lo cual el poseedor entra en relación.

Es importante recordar una advertencia que hace Putnam al presentar este experimento mental: sus resultados no cambian si se ubica a Oscar y a Oscar ${ }_{g}$ en, por ejemplo, el año 1625, cuando aún no se había descubierto la composición química del agua. No es necesario que Oscar sepa que la sustancia cuyas muestras causaron su concepto AGUA está compuesta por $\mathrm{H}_{2} \mathrm{O}$. Sólo es necesario que la sustancia que causó su concepto AGUA de hecho esté compuesta por $\mathrm{H}_{2} \mathrm{O}$. Incluso si Oscar y Oscar ${ }_{\mathrm{g}}$ hubieran vivido en 1625, si suponemos que las clases naturales eran las mismas en ese entonces (y es necesario suponerlo, ya que las clases naturales no cambian), entonces el experimento sigue funcionando: los contenidos mentales de Oscar y Oscar $_{g}$ son diferentes, y esto es gracias a que son el resultado de relaciones causales con sustancias diferentes, aunque ellos no reconozcan esas sustancias como

${ }^{4} \mathrm{El}$ experimento mental de las Tierras gemelas fue propuesto por Putnam para sostener el externalismo acerca del contenido lingüístico, pero es generalmente aceptado como un argumento para el externalismo acerca del contenido mental. 
clases naturales. Tener un concepto de una clase natural no equivale a tener un concepto como una clase natural (Fodor, 1998).

\section{Detectores y semántica informacional}

Habiendo expuesto las características y problemas de una teoría causalinformacional del contenido, estamos en condiciones de evaluar el éxito del empirismo de conceptos para dar cuenta del contenido intencional a través de un relato de este tipo. Recordemos que esta teoría propone identificar a los conceptos con detectores, es decir, con entidades estructuradas que reaccionan a determinados rasgos perceptibles.

Sostendremos que el tipo de vehículos que el empirismo de conceptos propone es incompatible con la semántica informacional que quiere sostener. Nos referiremos, en primer lugar, a las dificultades de los conceptos entendidos como detectores para explicar las representaciones erradas sin caer en el problema de la disyunción. Mostraremos cómo los detectores no son suficientes para aplicar la teoría semántica que Prinz propone para resolver este problema. En segundo lugar, presentaremos un argumento para mostrar que la determinación del contenido por medio de nada más que detectores de rasgos perceptibles no puede explicar el contenido de los conceptos de clase natural de manera externalista.

IV.1 Detectores, determinación del contenido y error La caracterización que hace Prinz de los conceptos, al proponer una semántica informacional, debe enfrentarse al desafío de explicar el error representacional sin caer en el problema de la disyunción. Tomemos, por ejemplo, el concepto CABALLO. Según el empirismo de conceptos, los conceptos son detectores de rasgos perceptibles, por lo que el contenido de este concepto estaría dado por la correlación entre casos de ciertos rasgos perceptibles y casos de activación del detector que reacciona a esos rasgos. Entonces, tener el concepto CABALLO no sería más que tener una representación perceptiva que reacciona a ese conjunto de rasgos. Ahora imaginemos una situación en la que este concepto está errado, digamos, cuando el concepto se activa ante la presencia de una vaca. Lo que ocurre, diríamos, es que el detector se activó ante la presencia de esa vaca, causando una ocurrencia de CABALLO. Pero esta representación no es el detector VACA, es decir, no debería reaccionar a vacas. ¿Cómo es posible entonces que se active ante la presencia de una vaca? Podríamos decir que esto se debe a que el detector CABALLO reacciona a los mismos rasgos que el detector VACA. Pero según esta teoría un concepto es un detector. Y un detector, podríamos decir, se individua según los rasgos que detecta. Si el detector 
CABALLO recoge los mismos rasgos que el detector VACA, entonces son el mismo detector. Y, si los conceptos no son más que detectores, entonces el concepto CABALLO y el concepto VACA son el mismo concepto, a saber, el concepto cuyo contenido es 'caballo o vaca'. Y no puede tener ese contenido disyuntivo, ya que lo que queríamos explicar en un principio era la activación errada del concepto CABALLO que se dispara ante una vaca.

Como vemos, el empirismo de conceptos no está exento de tener que lidiar con el problema de la disyunción. Prinz advierte esto y propone una solución inspirada en la salida ofrecida por Dretske expuesta más arriba. Sin embargo, también advierte que esta solución es objeto de las críticas de Fodor por estar formulada en subjuntivo (Prinz, 2002, pp. 249-250). Recordemos que Dretske propone distinguir entre los que ocurre en el período de aprendizaje de un concepto y el período posterior en el que el concepto ya fue aprendido. El error se explica porque un concepto R con contenido A puede ser causado por instancias de $\mathrm{B}$ en el período posterior al aprendizaje y resultar errado, siempre y cuando en el período de aprendizaje haya sido causado sólo por instancias de A. El problema del subjuntivo aparece cuando se considera que, sin embargo, si hubieran aparecido instancias de B en el período de aprendizaje entonces habrían causado también ocurrencias de $\mathrm{R}$, lo cual nos devuelve al problema del que habíamos partido. Prinz propone entonces eliminar el subjuntivo y apelar a una determinación del contenido por la causa incipiente actual. El contenido de un concepto está determinado por la propiedad cuya instancia causó la primera ocurrencia de ese concepto. Además, esa propiedad debe ser la que de hecho causó esa primera aparición del concepto, y no otra que lo habría causado si hubiera estado presente. Sin embargo, es difícil determinar cuál de todas las propiedades que posee un objeto es la que constituye el contenido del concepto formado en la experiencia de ese objeto. Esto se conoce como el qua problem $^{5}$. Para evitarlo, Prinz combina la teoría de la causa incipiente con la de covariación. La covaríación entre instancias de un concepto R y de alguna(s) propiedad(es) determina los potenciales contenidos de R. Ese conjunto de potenciales contenidos es restringido por la causa incipiente. De manera que $\mathrm{R}$ tiene el contenido $\mathrm{S}$ si:

1) instancias de $S$ covarían nomológicamente con instancias de $R ; y$

2) una instancia de $S$ fue la causa incipiente de R. (Prinz, 2002, p. 251)

${ }^{5}$ El qua problem es un problema de las teorías causales de la referencia introducido por Devitt y Sterelny (1999). El problema consiste en la dificultad para descubrir en virtud de cuál de las propiedades de un objeto se formó el contenido que resultó de la interacción causal perceptiva con él. 
De esta manera, se explicaría el error sin caer en contenidos disyuntivos. Supongamos que un agente tiene el concepto CABALLO y lo aplica a caballos, pero, algunas veces, lo aplica también, incorrectamente, a vacas. En ese caso, según la propuesta de Prinz, los contenidos potenciales del concepto son 'caballo' y 'vaca', ya que covaría con instancias de ambos. Entonces, para determinar mejor el contenido, recurrimos a la causa incipiente, y encontramos que la primera instancia del concepto en cuestión fue causada por un caballo. Así, restringimos el contenido a 'caballo' y podemos explicar que la aplicación del concepto a vacas sea un error. Como lo que estamos considerando es la propiedad del objeto que en esa experiencia de hecho causó el concepto por primera vez, no importa qué habría sucedido si la experiencia hubiera sido de una vaca. Lo único que importa es que el concepto de hecho fue causado por un caballo y, por lo tanto, su contenido es 'caballo'. Esta propuesta semántica híbrida que recurre a las relaciones de covariación tanto como a la causa incipiente vincularía las representaciones con propiedades del mundo, evitando caer en un verificacionismo.

Según el empirismo de conceptos, cuando se adquiere un concepto, lo que se adquiere es un mecanismo de detección. Cuando el organismo de nuestro ejemplo adquiere el concepto CABALLO, adquiere un detector para caballos. El detector CABALLO detecta caballos gracias a su estructura interna compuesta por reactores a rasgos perceptibles que los caballos (o algunos caballos, o la mayoría de los caballos) tienen. Este detector, probablemente, tiene partes en común con un detector para vacas, lo cual explicaría que reaccione, algunas veces, a vacas. Incluso, el detector para vacas podría estar compuesto por partes cuyo contenido sea exactamente el de las partes del detector para caballos. En ese caso, el detector para vacas tendría, por sus covariaciones con propiedades, los contenidos potenciales 'vaca' y 'caballo' pero su causa incipiente sería la interacción con una vaca, por lo cual su contenido sería vaca. El organismo en cuestión tendría dos detectores con las mismas partes significativas que tendrían contenidos diferentes.

Sin embargo, consideramos que esto no funciona como una explicación satisfactoria del contenido de estos conceptos. Si los conceptos son detectores, los detectores son entidades estructuradas cuyas partes tienen contenido, y los contenidos de las partes de un detector hacen que éste actúe como actúa, entonces es difícil pensar cómo dos detectores compuestos por las mismas partes significativas pueden no ser el mismo detector. Y, sin embargo, la teoría semántica de Prinz no impide que lleguemos a casos como este. 
Por otro lado, observamos que el empirismo de conceptos hace que sean posibles, además, escenarios como el siguiente: imaginemos un organismo $O$ cuya primera instancia del concepto CABALLO es causada, en un tiempo $t_{1}$, por cierto caballo $c_{1}$. Posteriormente, en un momento $t_{2}, O$ se encuentra con $\mathrm{c}_{2}$, y este encuentro causa nuevamente la activación de CABALLO. Pero $\mathrm{c}_{2} \mathrm{y} \mathrm{c} 1$ tienen algunas diferencias y no son muy similares en cuanto a sus propiedades perceptibles, a pesar de que ambos son caballos. Entonces, O debió modificar el mecanismo de detección para caballos, de manera que se active para $\mathrm{c}_{2}$ tanto como para $\mathrm{c}_{1}$. Además, $\mathrm{O}$ a veces se equivoca, ya que la vaca $v_{1}$ con frecuencia activa el concepto CABALLO. Si esto es así, ¿en qué sentido podemos decir que se activó el mismo concepto para $\mathrm{c}_{1} \mathrm{y}$ para $\mathrm{c}_{2}$ ? El detector cambió y, si el concepto es el detector, entonces éste también cambió. Esto resultaría en que $\mathrm{O}$ tenga un concepto para 'caballo' en $t_{1}$ y otro concepto diferente para la misma propiedad en $t_{2}$. Es decir, que los conceptos no serían públicos, ya que el mismo organismo en diferentes momentos no podría compartir el mismo concepto consigo mismo. Es fácil ver cómo también el mismo concepto variaría entre diferentes organismos. Collin Rice (2013) señala un problema similar con la determinación del contenido en la teoría de proxytipos: si el contenido está dado por aquella propiedad que covaría con la activación de un concepto, y esta activación consiste en el uso de una representación perceptiva (el proxytipo), entonces la misma propiedad podría causar contenidos diferentes, ya que una instancia de tal propiedad será detectada gracias a un proxytipo y otra instancia de la misma propiedad podría necesitar otro proxytipo (p.574). Esto es así, porque distintas instancias de la misma propiedad difieren, con frecuencia, en sus propiedades perceptivas. Pero como los conceptos no son más que detectores para propiedades perceptivas, entonces objetos con propiedades perceptivas diferentes covariarán con activaciones de representaciones diferentes $y$, por lo tanto, caerán necesariamente bajo conceptos diferentes.

Prinz puede dar una respuesta a este problema: un concepto, si bien no es más que un detector, no necesita detectar siempre exactamente los mismos rasgos. El sujeto, al estar en presencia de un objeto que cae bajo cierto concepto, incorpora la representación perceptiva de ese objeto a una red de representaciones que corresponde al concepto adecuado. Cuando ese sujeto necesita usar el concepto, usa una de las representaciones de la red de ese concepto, la que sea más apta para representar a toda la red y la que resulte más útil en ese contexto: el proxytipo. Prinz acepta que los proxytipos son flexibles (2002, pp.149-150), es decir, que variarán de acuerdo al contexto y a las experiencias que el sujeto tenga con instancias del concepto. De esta manera, el concepto CABALLO formado por la representación causada 
por $\mathrm{c}_{1}$ puede modificarse ante la presencia de $\mathrm{c}_{2}$, ya que la representación causada por $\mathrm{c}_{2}$ se incorpora a la red CABALLO modificando, tal vez, el conjunto de rasgos relevantes para reconocer caballos y el proxytipo disponible para la próxima aplicación del concepto CABALLO. Si esto es así, sin embargo, no está claro por qué no podría suceder algo similar con la representación causada por la vaca $\mathrm{v}_{1}$. Esta representación causó la activación del detector CABALLO, ¿no podría decirse, entonces, que se incorporó a la red correspondiente a ese concepto? ¿En este caso, el concepto CABALLO se modificaría para incluir ciertas vacas? La flexibilidad de los conceptos debería estar restringida de manera que esto no pueda ocurrir, pero la formulación que hace Prinz de este rasgo de los conceptos no incluye esta restricción. La limitación parece estar en la propiedad en el mundo que de hecho está presente cuando se forma la representación: la presencia de v1 no causa una modificación en el concepto CABALLO porque se trata de una vaca, y el concepto CABALLO fue causado por un caballo. La introducción de la causa incipiente en la semántica parece resolver este problema. Pero, si sólo entran en juego las propiedades perceptibles y la capacidad del concepto de detectarlas, no puede explicarse la determinación del contenido de esa manera, sólo puede explicarse que el contenido del concepto sea el caballo que lo causó y otros objetos lo suficientemente parecidos a ese caballo en cuanto a sus propiedades aparentes. Como también muestra Rice (2013), la causa incipiente sólo restringe aún más el contenido posible del concepto, ya que el proxytipo generado en el primer encuentro con una instancia de la propiedad no podría ser usado para detectar instancias posteriores que tengan propiedades perceptibles diferentes (p.575). La apelación a la causa incipiente no nos permite recurrir a la flexibilidad de los proxytipos. Entonces, si el concepto de hecho se activa en presencia de $\mathrm{v}_{1}$, tenemos que aceptar que fue aplicado correctamente, porque reaccionó a un objeto cuyas propiedades perceptibles superaron el umbral de detección. De la misma manera, la no activación de CABALLO ante un caballo atípico tampoco sería un problema, pues este caballo no comparte sus propiedades perceptivas con el caballo que causó el concepto y, por lo tanto, no entraría en la extensión del concepto. El concepto CABALLO, en ambos casos, está detectando lo que debe detectar (es decir, los rasgos perceptibles que poseen algunos caballos) pero no está indicando lo que debe indicar. Como el empirismo de conceptos sólo tiene en cuenta a los conceptos como detectores, no puede dar cuenta del error.

Notemos que estos problemas no surgen de sostener que los detectores intervienen en la activación de un concepto, sino de reducir los conceptos a los detectores. Prinz mismo define a los detectores como entidades que 
median entre los indicadores y las propiedades que éstos indican. Y son buenas entidades para postular cuando se les atribuye esa función: la de un mediador. Pero al eliminar los indicadores, ya no hay nada que mediar. Fodor (1984), al explicar el problema del error en las representaciones, se refiere a los detectores:

[S]uponga que $\mathrm{M}$ es un mecanismo cuya función es generar instancias de la representación tipo $\mathrm{R}$ en, y sólo en, instancias de la situación tipo $\mathrm{S}$. M media la relación causal entre Ss y Rs. Entonces, podemos decir que las ocurrencias de R producidas por M son irregulares (wild) cuando M está funcionando de manera anormal (pp. 247-248).

Como podemos ver, el mecanismo $\mathrm{M}$ es el detector de Prinz. La semántica causal-informacional no sólo incluye a los detectores en su explicación, sino que les da un lugar muy importante a la hora de dar cuenta del error. Cuando se produce una representación errada, lo que falla es precisamente el detector. Esto es inteligible si el detector es un medio para que se active el concepto ante la propiedad correspondiente, pero no lo es si el concepto es el detector. De la misma manera, la propuesta semántica de Prinz que combina causas incipientes actuales con relaciones de covariación entre propiedades y conceptos podría funcionar para determinar el contenido de los conceptos si estos fueran indicadores, pero es incompatible con la descripción de los conceptos que el mismo autor hace. La identificación de conceptos con detectores no permite explicar las representaciones erradas porque no conectan a los contenidos de los conceptos con propiedades del mundo, sino sólo con sus rasgos perceptibles. Entonces, que el detector falle implica que el contenido falla. ¿Y qué significaría que el contenido de un concepto falle? El contenido está dado por la propiedad a la cual el concepto debe reaccionar, aunque a veces no lo haga por fallas en el mecanismo de detección, y decir que el concepto debe reaccionar a cierta propiedad es decir que de hecho lo hace en circunstancias normales. Pero, para el empirismo de conceptos, las fallas en el mecanismo de detección son fallas en el concepto mismo, es decir, en su contenido. Y la única manera en la que entendemos esta falla del contenido es como la aceptación de cierto contenido en circunstancias anormales además del contenido que el concepto tiene en circunstancias normales. Pero si el contenido de un concepto incluye tanto las propiedades que producen sus ocurrencias en circunstancias normales como las que las producen en circunstancias anormales, entonces caemos nuevamente en el problema de la disyunción. 
Los detectores por sí mismos, como mostramos, no son suficientes para explicar cómo se determina el contenido intencional de un concepto. El recorte conceptual que hace Prinz parece resultar en una teoría incapaz de satisfacer los desiderata que el mismo autor persigue.

\section{IV.2 Detectores y clases naturales}

Existen conceptos cuyo contenido no es meramente una propiedad, sino que es un tipo de sustancia que tiene una esencia oculta que la hace ser lo que es. Estas sustancias son las clases naturales. Como vimos, este es el caso del agua, cuya esencia consiste en sus composición química: $\mathrm{H}_{2} \mathrm{O}$. Esta esencia es oculta, ya que no es accesible por las propiedades fenoménicas de la sustancia. En el experimento mental de las Tierras gemelas, se intentó poner en evidencia que podría existir otra sustancia con las mismas propiedades fenoménicas que el agua, pero que, si ésta no está compuesta por $\mathrm{H}_{2} \mathrm{O}$, entonces no es agua. Y no sólo eso, sino que, a pesar de tener las mismas propiedades fenoménicas que el agua, si no está compuesta por $\mathrm{H}_{2} \mathrm{O}$, entonces causa un contenido mental que no refiere al agua.

Como mencionamos, el paradigma en semántica para clases naturales es el externalismo: la doctrina que sostiene que los contenidos mentales están determinados en alguna medida por sus relaciones con sus referentes, y no por sus propiedades intrínsecas. El contenido de los conceptos de clase natural está determinado por las propiedades que la constituyen como clase, es decir, su esencia oculta, incluso si el agente poseedor del concepto no conoce esta esencia oculta. Como muestra el experimento de Putnam, incluso los organismos que se encontraron con el agua antes de que su composición química fuera descubierta adquirieron, a causa de ese encuentro, un concepto que refería al agua, esto es, a $\mathrm{H}_{2} \mathrm{O}$. Y no sólo eso: un organismo que tuviera el concepto AGUA, causado por muestras de agua, que viajara a la Tierra Gemela y se encontrara con muestras de agua ${ }_{\mathrm{g}}$ podría equivocarse y juzgar

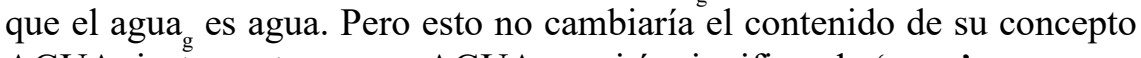
AGUA, justamente porque AGUA seguiría significando 'agua' es que sus pensamientos estarían errados y podrían ser corregidos posteriormente al descubrir la composición química del agua ${ }_{g}$

Según el empirismo de conceptos, Óscar, que vive en la Tierra y tiene el concepto AGUA, tiene nada más que un detector para el agua. En cambio, alguien que vive (y ha vivido siempre) en la Tierra Gemela, como Oscar ${ }_{g}$, tiene el concepto AGUA $_{G}$, es decir, tiene un detector para el agua $_{\mathrm{g}}$. Estos detectores reaccionan a los rasgos perceptibles del agua y del agua $_{\mathrm{g}}$, respectivamente. Pero, como fue mencionado en la exposición del experimento mental, estos rasgos son los mismos para el agua y para el agua, 
ya que sus propiedades fenoménicas son idénticas y se diferencian sólo por su composición química que está oculta y requiere de alguna actividad científica para ser descubierta. Entonces, resulta que el detector para agua y el detector para agua ${ }_{\mathrm{g}}$ son el mismo detector. Si se acepta la relación de identidad entre detectores y conceptos, no puede haber diferencia entre el concepto AGUA y el concepto AGUA $_{G}$. Los pensamientos de Oscar acerca del agua tendrían las mismas condiciones de corrección que los pensamientos de Oscar $_{\mathrm{g}}$ acerca del agua $_{\mathrm{g}}$. Es decir, que tendrían el mismo contenido. Pero, como sabemos, las sustancias que causan esos estados mentales no son idénticas. De la misma manera, si Oscar viajara a la Tierra Gemela, su detector para muestras de agua se activaría ante la presencia de agua ${ }_{\mathrm{g}}$, aunque tarde o temprano sabría que hay dos sustancias de naturaleza diferente y adquiriría el nuevo concepto $\mathrm{AGUA}_{\mathrm{G}}$. Ese nuevo concepto, sin embargo, consistiría en el mismo mecanismo de detección de rasgos perceptibles

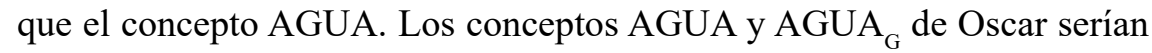
el mismo concepto.

Prinz explicaría la diferencia entre los conceptos AGUA Y AGUA mediante la teoría híbrida del contenido antes expuesta: la diferencia de contenido entre estos conceptos se explicaría porque el primero fue causado, en su primera ocurrencia, por una muestra de agua, mientras que el segundo fue causado por primera vez por una muestra de agua. Para conceptos de clase natural, además, Prinz adopta una posición que se deriva de la teoría lockeana y propone que la referencia de estos conceptos es doble. Por un lado, el concepto tiene un contenido nominal que consiste en aquellas propiedades perceptibles que reconocemos como identificando el concepto; $\mathrm{y}$, por otro lado, el concepto tiene un contenido real que refiere a la esencia de aquello a lo que el concepto refiere (Prinz, 2002, p. 277). En el caso del concepto AGUA, éste referiría tanto a las propiedades fenoménicas, como ser transparente, carecer de sabor, etc., como a su esencia, que consiste en estar compuesta por $\mathrm{H}_{2} \mathrm{O}$.

Esta teoría de la referencia doble, sin embargo, no parece dar muchas respuestas. Si bien Prinz afirma que los conceptos refieren de forma externalista, no es capaz de explicar esta afirmación en el marco de su teoría. Los conceptos entendidos como meros mecanismos de detección de rasgos perceptibles sólo parecen explicar el contenido nominal. Los proxytipos son, según el empirismo de Prinz, las únicas representaciones que intervienen en el uso de conceptos y son representaciones puramente perceptivas. De esta manera, podemos entender el papel del contenido nominal para individuar estas representaciones y, por lo tanto, para individuar conceptos. Lo que Prinz no provee son las claves para entender cómo los conceptos podrían 
individuarse según su contenido real. De hecho, el autor propone el siguiente mapeo entre proxytipos y contenido:

Cada ocurrencia de un concepto es un proxytipo. Esas ocurrencias pueden ser individuadas en su tipo de esa manera, hay una identidad de tipos entre conceptos y proxytipos. Según sus tipos nominales, los conceptos son proxytipos. Pero esta identidad de tipos falla cuando las ocurrencias de conceptos son individuadas por sus contenidos reales. Distintos proxytipos pueden corresponder al mismo contenido real, y distintos contenidos reales pueden corresponder al mismo proxytipo (Prinz, 2002, p. 279)

El funcionamiento de los conceptos así explicado no es nuevo ni controversial: sabemos que la correspondencia de conceptos con referentes no se da unívocamente. La existencia de los llamados "casos Frege" nos exige reconocer este hecho y explicar, como apunta Prinz, la diferencia entre contenido cognitivo y contenido intencional. Pero esta diferencia no puede explicarse si los conceptos no son más que detectores de rasgos perceptibles. Pensemos en el caso de las Tierras gemelas: Si Oscar, que tiene el concepto AGUA, viajara a la Tierra Gemela y se encontrara con agua ${ }_{\mathrm{g}}$, no adquiriría un nuevo tipo de detector. El detector que Oscar ya tenía para agua funcionaría ahora también para agua ${ }_{\mathrm{g}} \mathrm{y}$, por lo que sabemos, podría ser exactamente igual al detector que tiene Oscar ${ }_{\mathrm{g}}$ causado por agua $\mathrm{g}_{\mathrm{g}}$. Es decir, que, más allá de que Oscar conozca el agua ${ }_{\mathrm{g}}$ podría tener el mismo concepto para esa sustancia que Oscar ${ }_{\mathrm{g}}$, quien conoce el agua . En estos casos, el entorno de Oscar y de Oscar $_{\mathrm{g}}$ no determina el contenido de sus estados mentales, pues entornos distintos generan el mismo contenido nominal. Prinz podría decir que, en realidad, el concepto AGUA de Oscar consiste en las representaciones perceptivas generadas por su interacción con el agua y que, entonces, la representaciones causadas por su encuentro con agua $\mathrm{g}_{\mathrm{g}}$ no podrían formar parte de esa red. Después de todo, como es evidente en la cita anterior, el autor reconoce que los conceptos, además de individuarse como tipos de proxytipos, pueden ser individuados por su contenido intencional. Pero, si consideramos a los conceptos como meros detectores, entonces no entendemos cómo opera esta restricción externalista, ya que las propiedades detectadas son exactamente las mismas para el agua y para el agua $\mathrm{g}_{\mathrm{g}}$.

Prinz pretende defender el externalismo alegando que las propiedades recogidas por los proxytipos son propiedades en el mundo. Si bien esto puede ser cierto, no es obvio y requiere de mayor fundamentación. Pero incluso si aceptáramos esto, no sería suficiente: conocer las propiedades perceptibles no nos garantiza conocer todas las propiedades en el mundo y, en particular, 
no nos garantiza conocer las esencias. El punto del experimento mental de Putnam es exactamente ese. Prinz sugiere que la coincidencia entre contenidos nominales y contenidos reales está garantizada por el hecho de que los primeros están diseñados para rastrear los segundos (p. 282). Sin embargo, su propuesta no explica cómo se da este rastreo. La capacidad de los proxytipos en tanto contenidos nominales para conectarnos con los contenidos reales parece estar dado por una "armonía mágica" (cf. De Rosa, 2005, p. 601). Consideramos que, de hecho, no puede explicarse cómo los detectores refieren a propiedades del mundo. Prinz propone una teoría informacional que correlaciona partes de detectores y rasgos perceptibles y, a la vez, una teoría informacional que correlaciona conceptos con propiedades en el mundo. Pero debe elegir cuál de estas constituye su teoría del contenido intencional. De otra manera, este contenido estará sobredeterminado, ya que resulta de dos mecanismos diferentes y nuestra única esperanza será confiar en que entre esos mecanismos exista una coincidencia milagrosa. Prinz parece dar prioridad a la opción más económica, es decir, a la correlación entre detectores y rasgos perceptibles pero, como vimos, ésta no es suficiente para dar cuenta del contenido intencional de una manera externalista.

\section{Conclusión}

Prinz, al introducir su empirismo de conceptos, establece los desiderata que toda teoría de conceptos debería satisfacer e intenta ofrecer una teoría que no sólo cumpla con estas pretensiones, sino que lo haga de la manera más económica posible. Sin embargo, el empirismo de conceptos falla en dar una explicación satisfactoria del contenido intencional, ya que la descripción que hace de los conceptos como meros detectores no es compatible con el tipo de relato semántico informacional que quiere sostener. El empirista debe elegir entre defender su pretensión de economía y sostener una semántica exitosa. Una vez más, la cruza de conceptos no atómicos con una semántica informacional engendra monstruos, criaturas explicativamente estériles.

\section{Referencias bibliográficas}

De Rosa, R. (2005). Prinz's problematic proxytypes. The Philosophical Quarterly, 55(221), 594-606.

Devitt, M., \& Sterelny, K. (1999). Language and Reality. Basil Blackwell. Dretske, F. (1981). Knowledge and the Flow of Information. MIT/Bradford Press. Fodor, J. (1984). Semantics, Wisconsin Style. Synthese, 59(3), 231-250. Fodor, J. (1987). Psychosemantics: The Problem of Meaning in the Philosophy of Mind. MIT/Bradford.

Fodor, J. (1994). The Elm and the Expert. MIT Press. 
Fodor, J. (1998). Concepts: Where Cognitive Science Went Wrong. Oxford University Press.

Grice, H. P. (1957). Meaning. The Philosophical Review, 66(3), 377-88.

Markman, A. B., \& Stilwell, C. H. (2004). Concepts á la modal: An extended review of Prinz's Furnishing the mind. Philosophical Psychology, 17(3), 391-401. https://doi.org/10.1080/0951508042000286749

Prinz, J. (2002). Furnishing the Mind: Concepts and their Perceptual Basis. MIT Press.

Putnam, H. (1975). The Meaning of Meaning. Philosophy of Science, 7, 215-271. Rice, C. (2013). Concept empiricism, content, and compositionality. Philosophical Studies, 162(3), 567-583.

Skidelsky, L. (2011). La importancia de un buen transporte: la distinción conceptualno conceptual y el empirismo de conceptos. Analítica, (5), 29-60. 
